This is a postprint version of the following published document:

Gonzalo, Jesús; Lee, Tae-Hwy; Yang, Weiping. Permanent and transitory components of GDP and stock prices: further analysis. Macroeconomics and Finance in Emerging Market Economies, vol.1, issue 1, march 2008, p. 105-120. Avalaible in: http://dx.doi.org/10.1080/17520840701864955

(C)Taylor \& Francis 


\title{
Permanent and transitory components of GDP and stock prices: further analysis
}

\author{
Jesús Gonzalo ${ }^{\mathrm{a}}$, Tae-Hwy Lee ${ }^{\mathrm{b} *}$ and Weiping Yang ${ }^{\mathrm{c}}$ \\ ${ }^{a}$ Departamento de Economia, Universidad Carlos III de Madrid, Getafe, Madrid, Spain; ${ }^{b}$ Department \\ of Economics, University of California, Riverside, CA, USA; ${ }^{c}$ Capital One Financial Services, \\ Richmond, VA, USA
}

\begin{abstract}
Using the conventional VAR identification approach, Cochrane (Quarterly Journal of Economics 107: 241 65, 1994) finds that substantial amounts of variation in GDP growth and stock returns are due to transitory shocks. Following the common trend decomposition of King et al. (American Economic Review 81: 819 40, 1991), we show that Cochrane's results depend on the assumption of weak exogeneity of one of the variables with respect to the cointegration vector. When this assumption holds both approaches coincide. If not, the shocks Cochrane called transitory are not totally transitory. In this case, the conventional VAR approach with the assumption of the weak exogeneity may overstate the magnitude of transitory shocks and understate that of permanent shocks. We find that the permanent components of GDP and stock prices are much larger than those estimates of Cochrane, although substantial (but much smaller than in Cochrane 1994) variations in GDP growth and stock returns are attributed to transitory shocks.
\end{abstract}

Keywords: permanent components; transitory components; weak exogeneity; cointegration; VAR

\section{Introduction}

Cochrane (1994) finds strong evidence that substantial amounts of variation in GDP growth and stock returns are attributed to transitory shocks. He defines the transitory shock to the consumption-GDP system as a shock to GDP holding consumption constant so that the shock does not affect consumption contemporaneously. The facts that the consumption-GDP ratio does not forecast consumption growth and that consumption is nearly a random walk drive this definition.

Similarly, he defines transitory shocks to the dividend-price system as shocks to stock prices holding dividends constant so that the shock does not affect dividends contemporaneously. The facts that the dividend-price ratio may not forecast dividend growth and that dividend is nearly a random walk can justify this definition.

In this paper, we show that the transitory shocks defined by Cochrane do not have any long-run effect, if one of the variables is weakly exogenous with respect to the cointegration vector. However, if the assumption of weak exogeneity does not hold,

*Corresponding author. Email: taelee@ucr.edu 
shocks should be defined differently from Cochrane (1994). We define a permanent shock as a shock to the stochastic trend of a cointegrated system and a transitory shock as a shock orthogonal to the permanent shock. See Stock and Watson (1988) and King et al. (1991; KPSW hereafter). With this definition we ensure that in any cointegrated system the permanent shocks will have a long-run effect and the transitory shocks will not.

In general, if one of the error correction adjustment coefficients in a bivariate cointegrated system is zero, the conventional VAR method (CVAR hereafter) used by Cochrane and the KPSW method will produce the same type of shocks.

Cochrane (1994) studies situations where one of the error correction coefficients is insignificant. This may be implied by some economic theories such as the permanent income theory. However, if all of the error correction adjustment coefficients are significant, it would be interesting to compare the results from these two approaches (namely, CVAR and KPSW).

In this paper we found both error correction adjustment coefficients are significant in the consumption-GDP system and also in the dividend-stock price system. Accordingly, we found that the permanent components of GDP and stock prices are much larger than those estimates of Cochrane (1994), although substantial (but much smaller than in Cochrane 1994) variations in GDP growth and stock returns are attributed to transitory shocks.

The plan of the paper is as follows. In Section 2, we discuss the identification of permanent and transitory components in a bivariate cointegrated system, the impulse response function and variance decompositions associated with the shocks of each component. Section 3 deals with the identification when one of the variables is weakly exogenous with respect to the cointegrating vector. The empirical results are presented in Section 4. Section 5 concludes.

\section{Cointegrated vector autoregression model}

In this section we discuss the decomposition of a pair of cointegrated variables into permanent and transitory components. We compute the impulse response function and variance decompositions associated with the shocks of each component.

Let $X_{t}=\left(x_{t} y_{t}\right)^{\prime}$. If $X_{t}$ are cointegrated, it is well known that they admit the following common factor representation:

$$
X_{t}=X_{0}+\boldsymbol{\mu} t+\gamma f_{t}+\tilde{X}_{t}
$$

where $X_{0}=\left(\begin{array}{ll}x_{0} & y_{0}\end{array}\right)^{\prime}, \gamma=\left(\begin{array}{ll}\gamma_{1} & 1\end{array}\right)^{\prime}, f_{t}$ is a scalar integrated (denoted as $\left.I(1)\right)$ common permanent component, and $\tilde{X}_{t}=\left(\tilde{x}_{t} \tilde{y}_{t}\right)$ prime; is a vector of mean zero stationary (denoted as $I(0))$ transitory components. Cointegration implies that there exists a $2 \times 1$ vector $\boldsymbol{\beta}$ such that $\boldsymbol{\beta}^{\prime} \gamma=0$. Therefore, the cointegration error $z_{t}=\boldsymbol{\beta}^{\prime} X_{t}$ is $I(0)$.

As the permanent component is unobserved, we need some identification assumptions. Following KPSW, $f_{t}$ will be assumed to be a random walk. The innovation process of the permanent component, $\eta_{1 t}=f_{t}-f_{t}{ }_{1}=\Delta f_{t}$, will be called the permanent shock of the system $X_{t}$. The response of $X_{t}$ to the permanent shock $\eta_{1, t}{ }_{h}$ for $h=0,1,2, \ldots$ is denoted as $\partial X_{t} / \partial \eta_{1, t} h$. The impact multiplier at $h=0$ is $\partial X_{t} / \partial \eta_{1 t}$, and the long-run multiplier at $\mathrm{h} \rightarrow \infty$ is

$$
\lim _{h \rightarrow \infty} \partial X_{t} / \partial \eta_{1, t} h=\gamma
$$


From Granger's representation theorem, the pair of variables $X_{t}$ admits a vector error correction model (VECM) of infinite order, which can be approximated by

$$
\Delta X_{t}=\boldsymbol{\mu}+\Pi X_{t}{ }_{1}+A_{1} \Delta X_{t}{ }_{1}+\cdots+A_{k}{ }_{1} \Delta X_{t}{ }_{k+1}+\boldsymbol{\varepsilon}_{t}
$$

where $k$ is finite and $\varepsilon_{t}$ is a $2 \times 1$ vector white noise process with covariance matrix

$$
\Sigma_{\varepsilon}=\left(\begin{array}{cc}
\sigma_{1}^{2} & \sigma_{12} \\
\sigma_{12} & \sigma_{2}^{2}
\end{array}\right)
$$

As there exists only one common factor $f_{t}$, the cointegrating rank $r$ is equal to one, and thus the matrix II may be written as II $=\boldsymbol{\alpha} \boldsymbol{\beta}^{\prime}$, with both vectors $\boldsymbol{\alpha}=\left(\alpha_{1} \alpha_{2}\right)^{\prime}$ and $\boldsymbol{\beta}=\left(\beta_{1}\right.$ $\left.\beta_{2}\right)^{\prime}$ being $2 \times 1$ vectors. The vector $\alpha$ measures the speed of adjustment of the variables to the error correction term, and $\boldsymbol{\beta}$ is the cointegrating vector. Since $\boldsymbol{\beta}^{\prime} \boldsymbol{\gamma}=0$, then $\gamma_{1}=-\beta_{2}$ if $\beta_{1}$ is normalized at unity. Thus the long-run multiplier of $X_{t}$ to the permanent shock $\eta_{1 t}$ is $\gamma=\left(-\beta_{2} 1\right)^{\prime}$.

In Cochrane (1994), the consumption-GDP ratio and the dividend-stock price ratio are used, which implies the normalized cointegration vector is assumed to be $(1-1)^{\prime}$ (i.e. $\left.\beta_{2}=-1\right)$. This may be a relatively strong assumption. For instance, Barsky and De Long (1993) and Bansal and Lundblad (2002) show that the cointegration vector of dividend and stock price is not $(1-1)^{\prime}$. In this paper we do not impose known cointegration parameters and instead we estimate them. The null hypothesis that $\beta_{2}=-1$ is strongly rejected in our empirical section (see Table 5(b)).

We estimate the VECM in Equation (3), and then transform it into a vector moving average model (VMA) for $\Delta X_{t}$ :

$$
\Delta X_{t}=\boldsymbol{\mu}+C(B) \boldsymbol{\varepsilon}_{t}
$$

where $C(B)=\sum_{i=0}^{\infty} C_{i} B^{i}$ is a $2 \times 2$ matrix polynomial in the backshift operator $B$, with $C(0)=I$. As $\mathrm{C}(1)$ is of rank 1 , there exists a $2 \times 2$ vector $\boldsymbol{\delta}$ such that $C(1)=\boldsymbol{\gamma} \boldsymbol{\delta}^{\prime}$. Notice that the vector $\boldsymbol{\delta}$ is orthogonal to the adjustment coefficients $\boldsymbol{\alpha}$, that is, $\boldsymbol{\delta}^{\prime} \boldsymbol{\alpha}=0$, since $C(1) \boldsymbol{\alpha}=0$ and $\gamma \neq 0$. See Engle and Granger (1987, 256).

In order to identify the permanent and transitory shocks, we need to impose some identifying restrictions. Rewrite Equation (5) as

$$
\Delta X_{t}=\boldsymbol{\mu}+\Gamma(B) \boldsymbol{\eta}_{t}
$$

where

$$
\Gamma(B)=C(B) \Gamma_{0}
$$

with $\Gamma_{0}$ being a full rank matrix. The error term $\boldsymbol{\eta}_{t}=\left(\eta_{t} \eta_{2 t}\right)^{\prime}$ is a $2 \times 1$ vector white noise that satisfies

$$
\boldsymbol{\eta}_{t}=\Gamma_{0}{ }^{1} \boldsymbol{\varepsilon}_{t}
$$

and

$$
\Sigma_{\boldsymbol{\eta}}=E\left(\boldsymbol{\eta}_{t} \boldsymbol{\eta}_{t}^{\prime}\right)=\left(\begin{array}{cc}
\sigma_{\eta_{1}} & 0 \\
0 & \sigma_{\eta_{2}}
\end{array}\right) .
$$


We do not impose any restriction on the scale of $\Sigma_{\eta}$. From (7) and the assumption that $\eta_{1 t}$ is a permanent shock while $\eta_{2 t}$ is a transitory shock, the long-run multiplier matrix becomes

$$
\Gamma(1)=C(1) \Gamma_{0}=\boldsymbol{\gamma} \boldsymbol{\delta}^{\prime} \Gamma_{0}=(\boldsymbol{\gamma} \mathbf{0})
$$

Then

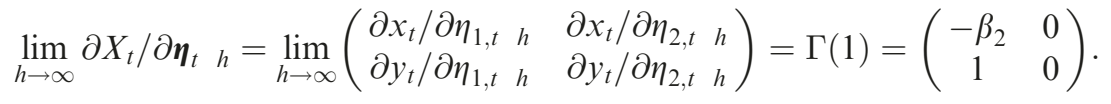

Using the expansion $\Gamma(B)=\Gamma(1)+\Delta \Gamma^{*}(B)$ in Equation (6), we have the common trend representation of Equation (1):

$$
X_{t}-X_{0}-\boldsymbol{\mu} t=\Delta{ }^{1} \Gamma(B) \boldsymbol{\eta}_{t}=\gamma \Delta{ }^{1} \eta_{1 t}+\Gamma^{*}(B) \boldsymbol{\eta}_{t}=\gamma f_{t}+\tilde{X}_{t}
$$

with $f_{t}=\Delta{ }^{1} \eta_{1 t}$ and $\tilde{X}_{t} \equiv \Gamma^{*}(B) \boldsymbol{\eta}_{t}$.

From the identifying restrictions (8), (9) and (10), the permanent $\left(\eta_{1 t}\right)$ and transitory $\left(\eta_{2 t}\right)$ shocks are easily obtained from the reduced form shocks of the estimated VECM in Equation (3). From (10)

$$
\Gamma_{0}=\left(\begin{array}{cc}
\frac{\theta}{\delta_{1}} & \delta_{2} \\
\frac{1-\theta}{\delta_{2}} & -\delta_{1}
\end{array}\right)
$$

and

$$
\Gamma_{0}^{1}=\left(\begin{array}{cc}
\delta_{1} & \delta_{2} \\
\frac{1-\theta}{\delta_{2}} & \frac{-\theta}{\delta_{1}}
\end{array}\right) .
$$

Therefore

$$
\eta_{1 t}=\delta_{1} \varepsilon_{1 t}+\delta_{2} \varepsilon_{2 t}
$$

and

$$
\eta_{2 t}=\frac{1-\theta}{\delta_{2}} \varepsilon_{1 t}-\frac{\theta}{\delta_{1}} \varepsilon_{2 t}
$$

where $\theta$ is chosen such that $E\left(\eta_{1 t}, \eta_{2 t}\right)=0$ that is

$$
\theta=\frac{\delta_{1}^{2} \sigma_{1}^{2}+\delta_{1} \delta_{2} \sigma_{12}}{\delta_{1}^{2} \sigma_{1}^{2}+2 \delta_{1} \delta_{2} \sigma_{12}+\delta_{2}^{2} \sigma_{2}^{2}} .
$$

Let the impulse responses of $x_{t}$ and $y_{t}$ to the permanent shock $\eta_{1, t} h$, that occurred at $h$ periods ago, be denoted as $\partial x_{t} / \partial \eta_{1, t}{ }_{h}$ and $\partial y_{t} / \partial \eta_{1, t}{ }_{h}$, respectively. These are given by the accumulated sum of the first column of $\Gamma$,'s that are obtained from the matrix lag polynomial $\Gamma(B)=\sum_{i=0}^{\infty} \Gamma_{i} B^{i}$. 
As the VECM can also be used for forecasting, we can compute the fractions of forecast error variances of $\Delta X_{t+h}$ due to the permanent innovation $\eta_{1 t}$, and they will yield interesting information about the relative importance of the permanent shock $\eta_{1 t}$ vs. the temporary shock $\eta_{2 t}$. They can be computed as follows. Let $V_{k}(h)$ be the fraction of $h$-step forecast error variance of $\Delta x_{k, t+h}, k=1,2$, where $x_{k t}$ is the $k$ th variable in $X_{t}=\left(x_{1 t} x_{2 t}\right)^{\prime}$, attributed to the innovation $\eta_{1 t}$ in the permanent stochastic trend. Then

$$
V_{k}(h)=\sum_{i=1}^{h} \gamma_{i, k 1}^{2} \sigma_{\eta_{1}}^{2} / M S E_{k}(h)
$$

where $\gamma_{\mathrm{i}, k 1}$ is the $(k, 1)$ element of $\Gamma_{\mathrm{i}}$ and the $M S E_{k}(h)$ is the $k$ th diagonal element $\operatorname{of} \operatorname{MSE}(h)=\sum_{i=0}^{h}{ }^{1} \Gamma_{i} \Sigma_{\eta} \Gamma_{i}^{\prime}=\sum_{i=0}^{h} C_{i} \Sigma_{\varepsilon} C_{i}^{\prime} . M S E(h)$ is the mean square error of the $h$-step forecast for $\Delta X_{t+h}$. Notice that even if only the first columns of $\Gamma_{i}$ 's are identified, $M S E(h)$ may be computed from $C_{i}$ 's. Following this notation, $V_{k}(1)$ denotes the fraction of the one-step forecast error variance for variable $k$ attributed to the permanent shock.

\section{When $x_{t}$ is weakly exogenous}

In this section, we examine the assumption made in Cochrane (1994). The variable $x_{t}$ is weakly exogenous with respect to the cointegrating vector $\beta$ if $\alpha_{1}=0$. In this case $\delta_{2}$ is also zero, since $\alpha_{2} \neq 0$ and $\boldsymbol{\delta}^{\prime} \boldsymbol{\alpha}=\delta_{2} \alpha_{2}=0$. From (10), (11) and (12), the permanent and transitory shocks are $\eta_{1 t}=\delta_{1 \varepsilon 1 t}$ and $\eta_{2 t}=\left(1 / \delta_{1}\right)\left(\varepsilon_{2 t}-\left(\sigma_{12} / \sigma_{1}^{2}\right) \varepsilon_{1 t}\right)$, respectively.

Cochrane (1994) uses the identification restrictions of the conventional VAR analysis. The orthogonalized and standardized shocks are $\boldsymbol{v}_{t}=\left(v_{1 t} v_{2 t}\right)^{\prime}=\Sigma_{\varepsilon}{ }^{1 / 2} \boldsymbol{\varepsilon}_{t}$, where

$$
\Sigma_{\varepsilon}^{1 / 2}=\left(\begin{array}{cc}
\sigma_{1} & 0 \\
\sigma_{12} / \sigma_{1} & \phi
\end{array}\right)
$$

is the Choleski square root of the covariance matrix $\Sigma_{\varepsilon}$ and $\phi \equiv\left(\sigma_{2}^{2}-\sigma_{12}^{2} / \sigma_{1}^{2}\right)^{1 / 2}$. It is straightforward to see that

$$
v_{1 t}=\left(1 / \sigma_{1}\right) \varepsilon_{1 t}
$$

and

$$
v_{2 t}=\frac{1}{\phi}\left(\varepsilon_{2 t}-\frac{\sigma_{12}}{\sigma_{1}^{2}} \varepsilon_{1 t}\right)
$$

For the KPSW approach, from $\boldsymbol{\gamma} \boldsymbol{\delta}^{\prime} \Gamma_{\mathrm{o}}=(\boldsymbol{\gamma} \mathbf{0})$ or $\boldsymbol{\delta}^{\prime} \Gamma_{\mathrm{o}}=\left(\begin{array}{ll}1 & \mathbf{0}\end{array}\right)$ with $\delta_{2}=0$, we have

$$
\Gamma_{0}=\left(\begin{array}{cc}
\frac{1}{\delta_{1}} & 0 \\
a & b
\end{array}\right)
$$

for any real numbers $a$ and $b$. If $b \neq 0$, then $\Gamma_{0}{ }^{1}$ exists and

$$
\Gamma_{0}{ }^{1}=\left(\begin{array}{cc}
\delta_{1} & 0 \\
-\frac{a}{b} \delta_{1} & \frac{1}{b}
\end{array}\right)
$$


Therefore

$$
\eta_{1 t}=\delta_{1} \varepsilon_{1 t}
$$

and

$$
\eta_{2 t}=-\frac{a}{b} \delta_{1} \varepsilon_{1 t}+\frac{1}{b} \varepsilon_{2 t}
$$

where $a$ is chosen such that $E\left(\eta_{1 t} \eta_{2 t}\right)=0$ for any $b \neq 0$, that is

$$
a=\frac{\sigma_{12}}{\delta_{1} \sigma_{1}^{2}} .
$$

Hence, when $x_{t}$ is weakly exogenous, the KPSW approach has the permanent shock

$$
\eta_{1 t}=\delta_{1} \varepsilon_{1 t}
$$

and the transitory shock

$$
\eta_{2 t}=\frac{1}{b}\left(\varepsilon_{2 t}-\frac{\sigma_{12}}{\sigma_{1}^{2}} \varepsilon_{1 t}\right)
$$

To compare the KPSW approach and the CVAR approach under weak exogeneity, we observe from (19), (26) that $v_{1 t}$ is proportional to $\eta_{1 t}\left(\right.$ as $\left.\delta_{1} \sigma_{1} v_{1 t}=\eta_{1 t}\right)$, and from (20), (27) that $v_{2 t}$ is proportional to $\eta_{2 t}$ (as $\phi v_{2 t}=b \eta_{2 t}$ ). Hence, under the weak exogeneity assumption that $\alpha_{1}=0$, the KPSW approach and the CVAR approach produce the same permanent and transitory shocks up to a constant multiplication.

The long-run multiplier of $v_{\mathrm{t}}$ is

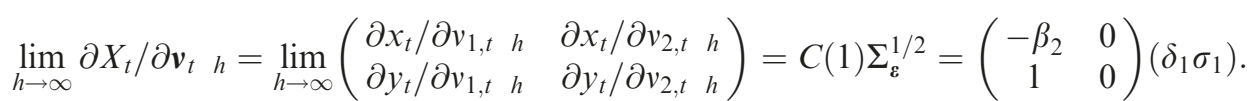

Comparing this with Equation (11) shows that the long-run multipliers of $\boldsymbol{\eta}_{t}$ and $\boldsymbol{v}_{t}$ are also the same up to a constant multiplication. Hence, the shocks $v_{1 t}$ and $v_{2 t}$ can be interpreted as a permanent and a transitory shock, respectively.

Therefore, we have shown that, if $\alpha_{1}=0$, the KPSW approach and the conventional VAR approach with the weak exogeneity assumption produce the same permanent and transitory shocks up to a constant multiplication. However, if none of the variables are weakly exogenous $\left(\alpha_{1} \neq 0\right.$ and $\left.\alpha_{2} \neq 0\right)$ the conventional VAR approach does not lead to the above interpretation. $v_{1 t}$ will no longer be a constant multiple of the permanent shock $\eta_{1 t}$, and $\eta_{2 t}$ will no longer be a transitory shock. The next section presents the various samples of the consumption-GDP system and the dividend-stock price system, for which there is generally no weak exogeneity and $\alpha_{1} \neq 0$ and $\alpha_{2} \neq 0$.

Remark 1. In order for CVAR and KPSW to produce the same type of shocks the variable that is weakly exogenous has to be ordered first in the VAR model. Ribba (1997) obtains 
the same type of result for CVAR and Blanchard and Quah (1989) orthogonalizations. For more than two variables see a very recent paper by Fisher and Huh (2007) where the results of Gonzalo and $\mathrm{Ng}$ (2001) about permanent and transitory shocks are particularized to the weak exogeneity case.

Remark 2. In general, common trends are only identified when there is only one permanent shock. If there is more than one and we want them to be orthogonal, we will always have to face the classical problem of the existence of multiple square roots of a covariance matrix. What is always identified, independent of the identification scheme, is the long-run impact of the permanent shocks $(\gamma)$.

Remark 3. Weak exogeneity is neither necessary nor sufficient for common trend identification. In the bivariate case as Crowder and Wohar (1998) show weak exogeneity would constitute an overidentifying condition and therefore testable as we do in the empirical part of this paper.

Remark 4. The identification problem of common trends can be overcome by identifying instead permanent components, for example, following Gonzalo and Granger (1995).

\section{Empirical results}

The data and the sample period used in this paper are summarized as follows. They are comparable to those of Cochrane (1994). As it is not possible to obtain exactly the same data-set used by Cochrane (1994), we have tried to get data that are as close as possible to his data. In this section, the following two systems are analysed.

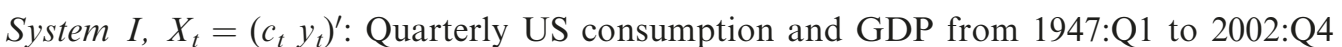
(sample size $n=228$ ) are obtained from Federal Reserve Economic Data (FRED) of St Louis FRB. We use natural logarithms of real GDP, denoted as $y_{t}$, and real consumption on non-durable goods and services, denoted as $c_{t}$, in 2000 dollars. In order to compare with the results of Cochrane (1994), we also use the data from FRED with the same sample period as in his paper, that is, quarterly US consumption and GDP from 1947:Q1 to 1989:Q3 $(n=171)$. The latter data-set is denoted as Sample (A) and the former with the sample period (1947:Q1 to 2002:Q4) is denoted as Sample (B). See Table 1.

Table 1. Data.

System I: Consumption GDP system (quarterly)

\begin{tabular}{lr}
\hline Sample (A) & \multicolumn{1}{c}{ Sample (B) } \\
\hline $\begin{array}{ll}\text { 1947:Q1 1989:Q3 } \\
n=171\end{array}$ & 1947:Q1 2002:Q4 \\
& $n=228$
\end{tabular}

System II: Dividend stock price system (yearly)

\begin{tabular}{lrr}
\hline Sample (C) & Sample (D) & Sample (E) \\
\hline 192788 & 18711988 & 18712002 \\
$n=62$ & $n=118$ & $n=132$ \\
\hline
\end{tabular}


System II, $X_{t}=\left(d_{t} p_{t}\right)^{\prime}$ : The natural logarithms of annual real dividends (denoted as $d_{t}$ ) and real stock prices (denoted as $p_{t}$ ) are used. The data was downloaded from the website of Robert Shiller at Yale. In order to compare with the results of Cochrane (1994), Sample (C) is the data with the same sample period as in Cochrane (1994), that is, from 1927 to $1988(n=62)$. The two expanded data-sets, Sample (D) from 1871 to $1988(n=118)$ and Sample (E) from 1871 to $2002(n=132)$, are also studied. See also Table 1.

\subsection{Empirical results for the consumption-GDP system}

First, we examine the cointegration in System I. The unit root tests in Table 2 and the cointegration tests in Table 3 indicate that System I is cointegrated for both Sample (A) and Sample (B). The lag length $k$ of the VECM in Equation (3) has been chosen by the Schwarz information criteria (SIC), that is, $k=2$ for System I.

We estimate the VECM by Johansen's method (1991). In Table 4, estimated $\hat{\boldsymbol{\alpha}}, \hat{\boldsymbol{\beta}}$ and $\hat{\boldsymbol{\delta}}$, are reported. $\beta_{1}$ is normalized at unity and $\alpha$ is re-scaled accordingly. According to the results in Tables 4 and 5(a), the null hypothesis that $\beta_{2}=-1$ is soundly rejected for both Sample (A) and Sample (B). Therefore, we estimate $\beta_{2}$ instead of assuming $\beta_{2}=-1$.

We then test for weak exogeneity by testing for $\alpha_{1}=0$ or for $\alpha_{2}=0$. The asymptotic tests in Table 5(a) strongly indicate that both $\alpha_{1}$ and $\alpha_{2}$ are significantly different from zero. The bootstrap tests that can be computed from the results in Table 4 (where the

Table 2. Tests for unit root.

\begin{tabular}{lccccc}
\hline \multicolumn{2}{l}{ System I: Consumption GDP system } & & \multicolumn{2}{c}{ Sample (B) } \\
\cline { 2 - 3 } \cline { 5 - 6 } Test & \multicolumn{2}{c}{ Sample (A) } & & $c_{t}$ & $y_{t}$ \\
\hline PP1 & 3.15 & 1.52 & & 0.14 & 0.75 \\
PP2 & 1.41 & 1.28 & & 1.28 & 0.61 \\
ADF1 & $2.25(2)$ & $1.19(1)$ & & $0.03(3)$ & $0.75(1)$ \\
ADF2 & $1.92(2)$ & $1.41(1)$ & & $1.61(3)$ & $0.77(1)$
\end{tabular}

System II: Dividend stock price system

\begin{tabular}{|c|c|c|c|c|c|c|}
\hline \multirow[b]{2}{*}{ Test } & \multicolumn{2}{|c|}{ Sample (C) } & \multicolumn{2}{|c|}{ Sample (D) } & \multicolumn{2}{|c|}{ Sample (E) } \\
\hline & $d_{t}$ & $p_{t}$ & $d_{t}$ & $p_{t}$ & $d_{t}$ & $p_{t}$ \\
\hline PP1 & 1.96 & 1.70 & 1.79 & 1.79 & 1.71 & 0.71 \\
\hline PP2 & 2.86 & 2.20 & $3.78^{*}$ & 2.95 & $3.99 *$ & 2.37 \\
\hline ADF1 & $1.90(0)$ & $1.65(0)$ & $1.98(0)$ & $1.84(0)$ & $1.93(1)$ & $0.74(0)$ \\
\hline ADF2 & $3.34(1)$ & $2.21(0)$ & $4.21 * *(1)$ & $2.85(0)$ & $4.47 * *(1)$ & $2.25(0)$ \\
\hline
\end{tabular}

Note: ADF and PP denote Augmented Dickey Fuller test and Phillips Perron test statistics, respectively. ADF1, PP1 are computed with a constant term, and ADF2, PP2 are with a constant and a linear trend. The numbers in parentheses for ADF are the number of lag augmentation, chosen using the SIC. The results do not change when the AIC is used. We report PPs with six non zero autocovariances. The critical values for both statistics, which are asymptotically equivalent, may be obtained from Fuller $(1976,373)$. The critical values of ADF1 are 3.45 and 2.87 at the $1 \%$ and $5 \%$ level, respectively. The critical values of ADF2 are 3.99 and 3.43 at the $1 \%$ and $5 \%$ level, respectively. The critical values of PP1 are 3.45 and 2.87 at the $1 \%$ and $5 \%$ level, respectively. The critical values of PP2 are 4.00 and 3.43 at the $1 \%$ and $5 \%$ level, respectively. $* *$ and $*$ denote the significance at $1 \%$ and $5 \%$ levels, respectively. 
Table 3. Tests for cointegration.

\begin{tabular}{lccccc}
\hline \multicolumn{2}{l}{ System I: Consumption GDP system } & & \\
\hline & \multicolumn{2}{c}{ Sample (A) } & & \multicolumn{2}{c}{ Sample (B) } \\
\cline { 2 - 3 } \cline { 5 - 6 } & Trace & $\lambda_{\max }$ & & Trace & $\lambda_{\max }$ \\
\hline$H_{0}: r=0$ & $40.85^{* *}$ & $38.51^{* *}$ & & $47.46^{* *}$ & $47.11^{* *}$ \\
$H_{0}: r \leq 1$ & 2.34 & 2.34 & & 0.35 & 0.35
\end{tabular}

System II: Dividend stock price system

\begin{tabular}{lccccccrr}
\hline & \multicolumn{2}{c}{ Sample $(\mathrm{C})$} & & \multicolumn{2}{c}{ Sample (D) } & & \multicolumn{2}{c}{ Sample (E) } \\
\cline { 2 - 3 } & Trace & $\lambda_{\max }$ & & Trace & $\lambda_{\max }$ & & Trace & $\lambda_{\max }$ \\
\hline$H_{0}: r=0$ & 12.37 & 10.78 & & $25.28^{* *}$ & $22.53^{* *}$ & & 14.04 & 13.59 \\
$H_{0}: r \leq 1$ & 1.59 & 1.59 & & 2.74 & 2.74 & & 0.17 & 0.17 \\
\hline
\end{tabular}

Note: $r$ denotes the rank of cointegration. The critical values for Johansen's trace statistic (Trace) and maximum eigenvalue statistic $\left(\lambda_{\max }\right)$ are obtained from Osterwald Lenum (1992). The critical values of trace statistics are 20.04 and 15.41 at the $1 \%$ and $5 \%$ level, respectively. For $\lambda_{\max }$, they are 18.63 and 14.07 , respectively. $* *$ and $*$ denote the significance at $1 \%$ and $5 \%$ levels. The lag value is chosen by using the SIC. Two lags for System I and one lag for System II are used throughout the paper.

Table 4. Some parameter estimates.

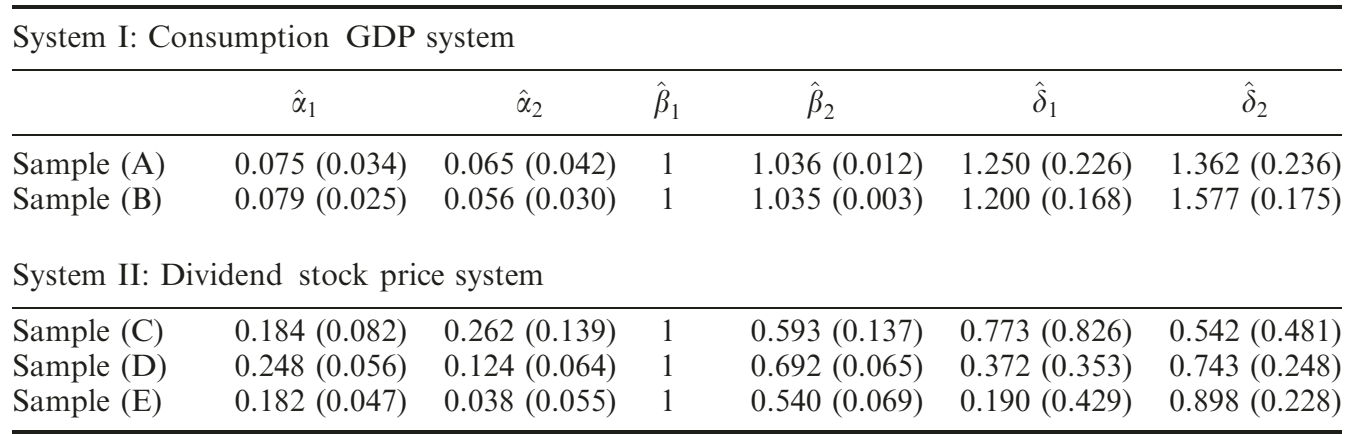

Note: All parameters are estimated from the vector error correction model (VECM) using the Johansen (1991) method. $\left(\alpha_{1} \alpha_{2}\right)^{\prime}$ is the speed of the error corrections and $\beta \quad\left(\beta_{1} \beta_{2}\right)^{\prime}$ is the cointegrating vector. $\hat{\beta}_{1}$ is normalized at unity. $\delta \quad\left(\delta_{1} \delta_{2}\right)^{\prime}$ is defined in $\eta_{1 t} \quad \delta^{\prime} \varepsilon_{t} \quad \delta_{1} \varepsilon_{1 t}+\delta_{2} \varepsilon_{2 t}$ so that $\delta$ is the weight vector to load the reduced form errors $\varepsilon_{t}$ on the permanent shock $\eta_{1 t}$. Bootstrap standard errors, shown in parentheses, are computed by the stationary bootstrap using 1000 replications.

stationary bootstrap of Politis and Romano 1994 is used to simulate 1000 bootstrap resamples) also indicate that $\alpha_{1}$ is significantly different from zero and $\alpha_{2}$ is marginally significantly different from zero. This is true for both Samples (A) and (B). Hence the weak exogeneity assumption of $\alpha_{1}=0$ (as imposed in Cochrane 1994) is invalidated for System I.

The impulse responses of $X_{t}$ to the permanent shock are reported in Table 6. We use the VMA model in Equation (5) of order 24. We also used the VMA models of orders 20, 36 and 40 , but the results were almost the same. The estimated responses $\partial X_{t} / \partial \eta_{1, t} h$ get closer to $\gamma$ as $h$ increases. Both series in each system complete the adjustment at the steady 
Table 5(a). Johansen test for the significance of parameters.

\begin{tabular}{lcccc}
\hline \multicolumn{2}{l}{ System I: Consumption } & GDP system & & \\
\hline & $\hat{\alpha}_{1}$ & $\hat{\alpha}_{2}$ & $\hat{\beta}_{1}$ & $\hat{\beta}_{2}$ \\
\hline Sample (A) & $0.075[0.00]$ & $0.065[0.02]$ & 1 & $1.036[0.00]$ \\
Sample (B) & $0.079[0.00]$ & $0.056[0.02]$ & 1 & $1.035[0.00]$ \\
& & & & \\
\multicolumn{2}{l}{ System II: Dividend stock price system } & & & \\
\hline Sample (C) & $0.184[0.17]$ & $0.262[0.19]$ & 1 & $0.593[0.00]$ \\
Sample (D) & $0.248[0.00]$ & $0.124[0.31]$ & 1 & $0.692[0.00]$ \\
Sample (E) & $0.182[0.00]$ & $0.038[0.72]$ & 1 & $0.540[0.00]$ \\
\hline
\end{tabular}

Note: All parameters are estimated from the VECM using the Johansen (1991) method. The asymptotic $p$ value of the Johansen test for the null hypothesis that each parameter is zero is shown in brackets.

Table 5(b). Johansen test for $H_{0}: \beta_{2}=1$.

System I: Consumption GDP system

\begin{tabular}{lc}
\hline Sample (A) & Sample (B) \\
\hline $31.82[0.000]$ & $46.04[0.000]$
\end{tabular}

System II: Dividend stock price system

\begin{tabular}{lcc}
\hline Sample $(\mathrm{C})$ & Sample (D) & Sample (E) \\
\hline $5.125[0.023]$ & $10.390[0.001]$ & $11.93[0.001]$ \\
\hline
\end{tabular}

Note: Johansen statistics for $H_{0}: \beta_{2} \quad 1$ are reported together with their asymptotic $p$ values in brackets.

state suggested by the estimated cointegrating vector reported in the last row of Table 6. As expected from the common stochastic trend representation in Equation (8), the longrun multiplier of the permanent shock is $\gamma$; that is, $\lim _{h \rightarrow \infty} \partial x_{t} / \partial \eta_{1, t h}=-\beta_{2}$ and $\lim _{h \rightarrow \infty} \partial y_{t} / \partial \eta_{1, t h}=1$ by normalization. In order to find the standard error for the impulse-response estimates, we also use the stationary bootstrap.

The estimated fractions of the forecast error variances of $\Delta X_{t+h}$ attributed to $\eta_{1 t}$ are presented in Table 7 ( $h$ is the forecast horizon in this case). The results show that the fraction of forecast error variance of GDP due to permanent shocks is slightly higher than that of consumption. In order to find the standard errors for the fraction estimates, we also use the stationary bootstrap.

In Table 8, the results of the variance decompositions of one-step forecast error using the CVAR method with $\alpha_{1}=0$ imposed (Panel B) and the results from Table 7 for $h=1$ using the KPSW method (Panel A) are presented. Because as mentioned earlier, it was not possible to get exactly the same data-set used by Cochrane (1994), we also copied Cochrane's results (1994) in Panel C of Table 8. For Sample (A), the CVAR method with the $\alpha_{1}=0$ restriction, $31 \%$ of the one-step ahead forecast error variance in quarterly GDP growth is due to permanent shocks. If the KPSW method is used the number is much bigger, $88 \%$. In Sample (B), with the CVAR method with $\alpha_{1}=0$ imposed, it is $30 \%$, while the KPSW method yields $91 \%$ of the one-step forecast error variance in the GDP growth 
Table 6. Impulse responses of $X_{t}$ to $\eta_{1, t-h}$.

\begin{tabular}{|c|c|c|c|c|}
\hline \multirow[b]{2}{*}{$h$} & \multicolumn{2}{|c|}{ Sample (A) } & \multicolumn{2}{|c|}{ Sample (B) } \\
\hline & $\partial c_{t} / \partial \eta_{1, t-h}$ & $\partial y_{t} / \partial \eta_{1, t-h}$ & $\partial c_{t} / \partial \eta_{1, t-h}$ & $\partial y_{t} / \partial \eta_{1, t-h}$ \\
\hline 0 & $0.266(0.416)$ & $0.490(0.298)$ & $0.235(0.366)$ & $0.456(0.251)$ \\
\hline 1 & $0.399(0.110)$ & $0.773(0.334)$ & $0.362(0.098)$ & $0.712(0.182)$ \\
\hline 2 & $0.491(0.329)$ & $0.905(0.250)$ & $0.453(0.288)$ & $0.840(0.203)$ \\
\hline 3 & $0.559(0.135)$ & $0.970(0.269)$ & $0.520(0.122)$ & $0.907(0.142)$ \\
\hline 4 & $0.613(0.266)$ & $1.000(0.206)$ & $0.575(0.232)$ & $0.943(0.165)$ \\
\hline 8 & $0.764(0.182)$ & $1.029(0.143)$ & $0.732(0.158)$ & $0.993(0.113)$ \\
\hline 12 & $0.862(0.102)$ & $1.032(0.107)$ & $0.838(0.113)$ & $1.011(0.081)$ \\
\hline 24 & $1.004(0.056)$ & $1.033(0.058)$ & $1.000(0.047)$ & $1.036(0.048)$ \\
\hline$\infty$ & $1.036(0.012)$ & 1.000 & $1.035(0.012)$ & 1.000 \\
\hline
\end{tabular}

System II: Dividend stock price system

\begin{tabular}{|c|c|c|c|c|c|c|}
\hline \multirow[b]{2}{*}{$h$} & \multicolumn{2}{|c|}{ Sample (C) } & \multicolumn{2}{|c|}{ Sample (D) } & \multicolumn{2}{|c|}{ Sample (E) } \\
\hline & $\partial d_{t} / \partial \eta_{1, t-h}$ & $\partial p_{t} / \partial \eta_{1, t-h}$ & $\partial d_{t} / \partial \eta_{1, t-h}$ & $\partial p_{t} / \partial \eta_{1, t-h}$ & $\partial d_{t} / \partial \eta_{1, t-h}$ & $\partial p_{t} / \partial \eta_{1, t-h}$ \\
\hline 0 & $0.583(0.167)$ & $1.014(0.241)$ & $0.544(0.130)$ & $1.074(0.089)$ & $0.408(0.138)$ & $1.028(0.074)$ \\
\hline 1 & $0.586(0.151)$ & $1.009(0.206)$ & $0.593(0.110)$ & $1.049(0.074)$ & $0.435(0.124)$ & $1.022(0.065)$ \\
\hline 2 & $0.588(0.139)$ & $1.006(0.177)$ & $0.626(0.095)$ & $1.033(0.062)$ & $0.457(0.112)$ & $1.017(0.058)$ \\
\hline 3 & $0.590(0.129)$ & $1.004(0.153)$ & $0.648(0.083)$ & $1.021(0.053)$ & $0.473(0.103)$ & $1.014(0.052)$ \\
\hline 4 & $0.591(0.122)$ & $1.002(0.134)$ & $0.663(0.073)$ & $1.014(0.046)$ & $0.486(0.095)$ & $1.011(0.047)$ \\
\hline 8 & $0.592(0.104)$ & $1.000(0.087)$ & $0.686(0.052)$ & $1.002(0.032)$ & $0.518(0.074)$ & $1.004(0.034)$ \\
\hline 12 & $0.593(0.097)$ & $1.000(0.067)$ & $0.691(0.046)$ & $1.001(0.029)$ & $0.531(0.063)$ & $1.002(0.029)$ \\
\hline 24 & $0.593(0.092)$ & $1.000(0.055)$ & $0.692(0.042)$ & $1.000(0.029)$ & $0.539(0.053)$ & $1.000(0.029)$ \\
\hline$\infty$ & $0.593(0.137)$ & 1.000 & $0.692(0.065)$ & 1.000 & $0.540(0.069)$ & 1.000 \\
\hline
\end{tabular}

Note: For each system there are two columns. Each column reports the impulse responses of the level series $x_{t}$ to the permanent shock $\eta_{1, t} h$ that occurred $h$ periods ago. The estimated long run multipliers of $X_{t}$ to the permanent shock are $\left(\hat{\beta}_{2} 1\right)^{\prime}$. These are the impulse responses of $X_{t}$ as $h \rightarrow \infty$, and are obtained from the estimated cointegrating vector $\left(1 \hat{\beta}_{2}\right)^{\prime}$ reported in Table 4 . Bootstrap standard errors, shown in parentheses, are computed by the stationary bootstrap using 1000 replications.

due to the permanent shock. A one-sided test based on stationary bootstrap is used to test whether the difference of these estimates in these two models is significant or not. Denote $V_{y}^{\mathrm{KPSW}}(1)$ as the fraction estimate for one-step forecast error variance of GDP due to permanent shocks by the KPSW method, and $V_{y}^{\mathrm{CVAR}}(1)$ as that for the CVAR model with $\alpha_{1}=0$ imposed. The null hypothesis of the test is $H_{0}: V_{y}^{\mathrm{KPSW}}(1)-V_{y}^{\mathrm{CVAR}}(1) \leq 0$. The $p$-values of the test are obtained by using bootstrap resampling with 1000 repetition times. For each resampled data, the two estimates are calculated and the bootstrap distribution of the test statistic is obtained. The test results for the two samples in Table 9 show that the permanent component of the GDP is significantly larger than those estimates of the CVAR or Cochrane (1994). ${ }^{1}$

The above result verifies our analysis in Section 3. If both adjustment coefficients are significant, permanent shocks in the KPSW model are different from that in the CVAR model. In the consumption-GDP system, both adjustment coefficients are significant, therefore, the results of the VECM and CVAR are different. The empirical results show that the CVAR approach underestimates the permanent shocks and overestimates the transitory shocks when the weak exogeneity assumption does not hold. 
Table 7. Fractions of forecast error variances attributed to $\eta_{1}$.

\begin{tabular}{|c|c|c|c|c|}
\hline \multirow[b]{2}{*}{$h$} & \multicolumn{2}{|c|}{ Sample (A) } & \multicolumn{2}{|c|}{ Sample (B) } \\
\hline & $\hat{V}_{c}(h)$ & $\hat{V}_{y}(h)$ & $\hat{V}_{c}(h)$ & $\hat{V}_{y}(h)$ \\
\hline 1 & $0.649(0.226)$ & $0.882(0.202)$ & $0.601(0.179)$ & $0.907(0.072)$ \\
\hline 2 & $0.696(0.225)$ & $0.881(0.127)$ & $0.660(0.177)$ & $\mathrm{x} 0.900(0.042)$ \\
\hline 3 & $0.714(0.214)$ & $0.884(0.095)$ & $0.684(0.169)$ & $0.903(0.043)$ \\
\hline 4 & $0.723(0.216)$ & $0.885(0.089)$ & $0.696(0.171)$ & $0.903(0.052)$ \\
\hline 8 & $0.735(0.212)$ & $0.885(0.083)$ & $0.711(0.169)$ & $0.904(0.063)$ \\
\hline 12 & $0.738(0.212)$ & $0.885(0.083)$ & $0.717(0.168)$ & $0.904(0.066)$ \\
\hline 24 & $0.741(0.212)$ & $0.885(0.083)$ & $0.721(0.168)$ & $0.904(0.067)$ \\
\hline
\end{tabular}

System II: Dividend stock price system

\begin{tabular}{|c|c|c|c|c|c|c|}
\hline \multirow[b]{2}{*}{$h$} & \multicolumn{2}{|c|}{ Sample (C) } & \multicolumn{2}{|c|}{ Sample (D) } & \multicolumn{2}{|c|}{ Sample (E) } \\
\hline & $\hat{V}_{d}(h)$ & $\hat{V}_{p}(h)$ & $\hat{V}_{d}(h)$ & $\hat{V}_{p}(h)$ & $\hat{V}_{d}(h)$ & $\hat{V}_{p}(h)$ \\
\hline 1 & $0.788(0.260)$ & $0.846(0.286)$ & $0.539(0.199)$ & $0.948(0.158)$ & $0.365(0.211)$ & $0.987(0.109)$ \\
\hline 2 & $0.769(0.259)$ & $0.832(0.286)$ & $0.515(0.198)$ & $0.942(0.160)$ & $0.356(0.210)$ & $0.987(0.110)$ \\
\hline 3 & $0.761(0.258)$ & $0.825(0.286)$ & $0.505(0.198)$ & $0.940(0.161)$ & $0.352(0.209)$ & $0.987(0.111)$ \\
\hline 4 & $0.757(0.257)$ & $0.823(0.286)$ & $0.500(0.198)$ & $0.939(0.162)$ & $0.349(0.208)$ & $0.986(0.112)$ \\
\hline 8 & $0.755(0.256)$ & $0.821(0.286)$ & $0.498(0.198)$ & $0.938(0.163)$ & $0.344(0.207)$ & $0.986(0.113)$ \\
\hline 12 & $0.755(0.256)$ & $0.821(0.286)$ & $0.497(0.197)$ & $0.938(0.164)$ & $0.343(0.207)$ & $0.987(0.113)$ \\
\hline 24 & $0.755(0.255)$ & $0.821(0.285)$ & $0.497(0.197)$ & $0.938(0.164)$ & $0.343(0.206)$ & $0.987(0.113)$ \\
\hline
\end{tabular}

Note: For each system there are two columns. $\hat{V}_{x}(h)$ denotes the estimated fractions of forecast error variance of $\Delta x_{t+h}$ attributed to $\eta_{1 t}$. Bootstrap standard errors, shown in parentheses, are computed by the stationary bootstrap using 1000 replications.

As we find $\alpha_{1} \neq 0$, the consumption is not a pure random walk. The result is in accordance with Jaeger (1992) who finds that consumption is not a pure random walk. The variation in consumption can be affected by transitory shocks if $\alpha_{1}$ and $\delta_{2}$ are not zero. Our results from the KPSW method show that a substantial amount of the forecast error variance for quarterly consumption growth is due to transitory shocks $-35 \%$ for Sample (A) and $40 \%$ for Sample (B). These numbers would be zero if the CVAR method with $\alpha_{1}=0$ were used.

\subsection{Empirical results for the dividend-stock price system}

For System II with dividend and stock price, Table 2 reports the unit root tests and Table 3 the cointegration tests. The lag length $k=1$ of the VECM in Equation (3) is selected by the SIC. In the three samples used, only Sample (D) demonstrates a strong cointegration relationship. The cointegration relation is insignificant or marginal for Samples (C) and (E). However, we proceed to estimate the VECM with the cointegrating rank $r=1$ for all three samples. In Table 4, estimated Table 4, estimated $\hat{\boldsymbol{\alpha}}, \hat{\boldsymbol{\beta}}$ and $\hat{\boldsymbol{\delta}}$ are reported. $\beta_{1}$ is normalized at unity and $\alpha$ is re-scaled accordingly. According to the results in Table 4 (using the bootstrap tests) and Table 5(a) (using the asymptotic tests), the null hypothesis that $\beta_{2}=-1$ is soundly rejected for all three samples and thus we estimate $\beta_{2}$ instead of assuming $\beta_{2}=-1$.

We then test for weak exogeneity by testing for $\alpha_{1}=0$ or for $\alpha_{2}=0$. The asymptotic tests in Table 5(a) for System II strongly indicate that $\alpha_{1} \neq 0$ while $\alpha_{2}$ may be 
Table 8. Decompositions of one step forecast error variances Table 4, estimated $\hat{V}(1)$ : comparisons.

\begin{tabular}{|c|c|c|c|c|c|c|}
\hline \multicolumn{7}{|c|}{ System I: Consumption GDP system } \\
\hline & \multicolumn{3}{|c|}{ Sample (A) } & & \multicolumn{2}{|c|}{ Sample (B) } \\
\hline & \multicolumn{2}{|c|}{$\Delta c_{t+1}$} & $\Delta y_{t+1}$ & \multicolumn{2}{|r|}{$\Delta c_{t+1}$} & $\Delta y_{t+1}$ \\
\hline \multicolumn{7}{|l|}{ Panel A. KPSW } \\
\hline Due to permanent shock & \multicolumn{2}{|c|}{0.65} & 0.88 & \multicolumn{2}{|r|}{0.60} & 0.91 \\
\hline Due to temporary shock & & & 0.12 & & 0.40 & 0.09 \\
\hline \multicolumn{7}{|c|}{ Panel B. CVAR imposing weak exogeneity } \\
\hline Due to permanent shock & \multirow{2}{*}{\multicolumn{2}{|c|}{$\begin{array}{l}1.00 \\
0.00\end{array}$}} & 0.30 & \multicolumn{2}{|r|}{1.00} & 0.30 \\
\hline Due to temporary shock & & & 0.70 & & 0.00 & 0.70 \\
\hline \multicolumn{7}{|c|}{ Panel C. Cochrane’s CVAR results imposing weak exog. } \\
\hline Due to permanent shock & \multirow{2}{*}{\multicolumn{2}{|c|}{$\begin{array}{l}1.00 \\
0.00\end{array}$}} & \multirow{2}{*}{\multicolumn{2}{|c|}{$\begin{array}{l}0.15 \\
0.85\end{array}$}} & & \\
\hline Due to temporary shock & & & & & & \\
\hline \multicolumn{7}{|c|}{ System II: Dividend stock price system } \\
\hline & \multicolumn{2}{|c|}{ Sample (C) } & \multicolumn{2}{|c|}{ Sample (D) } & \multicolumn{2}{|c|}{ Sample (E) } \\
\hline & $\Delta d_{t+1}$ & $\Delta p_{t+1}$ & $\Delta d_{t+1}$ & $\Delta p_{t+1}$ & $\Delta d_{t+1}$ & $\Delta p_{t+1}$ \\
\hline \multicolumn{7}{|l|}{ Panel A. KPSW } \\
\hline Due to permanent shock & 0.79 & 0.85 & 0.54 & 0.95 & 0.37 & 0.99 \\
\hline Due to temporary shock & 0.21 & 0.15 & 0.46 & 0.05 & 0.63 & 0.01 \\
\hline \multicolumn{7}{|c|}{ Panel B. CVAR imposing weak exogeneity } \\
\hline Due to permanent shock & 1.00 & 0.41 & 1.00 & 0.31 & 1.00 & 0.26 \\
\hline Due to temporary shock & 0.00 & 0.59 & 0.00 & 0.69 & 0.00 & 0.74 \\
\hline \multicolumn{7}{|c|}{ Panel C. Cochrane's CVAR results imposing weak exogeneity } \\
\hline Due to permanent shock & 1.00 & 0.45 & & & & \\
\hline Due to temporary shock & 0.00 & 0.55 & & & & \\
\hline
\end{tabular}

Note: This table shows the results comparable to those in Cochrane (1994, Tables I and II). His results are also copied in Panel C. As he reports only for $h \quad 1$, we report only for that in Panels A and B. Cochrane (footnote 5) also reports for private GNP for which the fraction of one step forecast error variance due to the temporary shock is 0.89. The results in Panel A are obtained from Table 7 for $h \quad 1$. The column under the heading $\Delta x_{t+1}$ reports the fraction of forecast error variance, $\operatorname{Var}\left(\begin{array}{cc}\Delta x_{t+1} & E_{t} \Delta x_{t+1}\end{array}\right)$ that is attributed to each shock.

insignificantly different from zero. The bootstrap tests that can be computed from the results in Table 4 also clearly indicate that $\alpha_{1}$ is significantly different from zero for all three samples, and that $\alpha_{2}$ is marginally significantly different from zero for Samples (C) and (D). Hence the weak exogeneity assumption of $\alpha_{1}=0$ (as imposed in Cochrane 1994) is invalidated for System II (as well as for System I).

The impulse responses of $X_{t}$ to the permanent shock are reported in Table 6. We use the VMA model in Equation (5) of order 24. The estimated responses $\partial X_{t} / \partial \eta_{1, t} h$ also get closer to $\gamma$ as $h$ increases. The impulse responses complete the adjustment at the steady state suggested by the estimated cointegrating vector. The standard error for the impulseresponse estimates are also obtained by stationary bootstrap.

The estimated fractions of the forecast error variances of $\Delta X_{t+h}$ attributed to the permanent shock $\eta_{1 t}$ presented in Table 7 show that they are slightly higher for stock price than for dividend.

In Table 8, the results of the variance decompositions of one-step forecast error using the CVAR method with $\alpha_{1}=0$ imposed (Panel B) and the results from Table 7 for $h=1$ using the KPSW method (Panel A) are presented. Because as mentioned earlier, it was not 
Table 9. Tests for comparing one step forecast error variances decompositions.

System I: Consumption GDP system

$\mathrm{H}_{0}: V_{y}^{\mathrm{KPSW}}(1) \leq V_{y}^{\mathrm{CVAR}}(1)$

\begin{tabular}{lc}
\hline Sample (A) Sample (B) \\
\hline 0 (A)
\end{tabular}

\begin{tabular}{ll}
\hline $0.57[0.042]$ & $0.61[0.014]$
\end{tabular}

System II: Dividend stock price system

$H_{0}: V_{p}^{\mathrm{KPSW}}(1) \leq V_{p}^{\mathrm{CVAR}}(1)$

\begin{tabular}{lll}
\hline Sample (C) & Sample (D) & Sample (E) \\
\hline $0.44[0.098]$ & $0.64[0.001]$ & $0.73[0.003]$ \\
\hline
\end{tabular}

Note: This table shows the difference in one step forecast error variance for KPSW and CVAR models. For System I, $V_{y}^{\mathrm{KPSW}}(1)$ denotes the fraction estimate for one step forecast error variance of GDP due to permanent shocks by the KPSW method, and $V_{y}^{\text {CVAR }}(1)$ denotes that for the CVAR model imposing $\alpha_{1}$ 0. For System II, $V_{p}^{\mathrm{KPSW}}(1)$ denotes the fraction estimate for one step forecast error variance of stock price due to permanent shocks by the KPSW method, and $V_{p}^{\mathrm{CVAR}}(1)$ denotes that for the CVAR model imposing $\alpha_{1} \quad 0$. The bootstrap $p$ values in brackets are obtained by using the stationary bootstrap with 1000 replications.

possible to get exactly the same data-set used by Cochrane (1994), we also copied Cochrane's results (1994) in Panel C of Table 8. For Sample (C), the CVAR method with the $\alpha_{1}=0$ restriction, $41 \%$ of the one-step ahead forecast error variance in quarterly GDP growth is due to permanent shocks. If the KPSW method is used the number is much bigger, $35 \%$. In Sample (D), with the CVAR method with $\alpha_{1}=0$ imposed, it is $31 \%$, while the KPSW method yields $95 \%$ of the one-step forecast error variance in stock price due to the permanent shock. In Sample (E), with the CVAR method with $\alpha_{1}=0$ imposed, it is $26 \%$, while the KPSW method yields $99 \%$ of the one-step forecast error variance in the stock price due to the permanent shock. A one-sided test based on stationary bootstrap is used to test whether the difference of these estimates in these two models is significant or not. Denote $V_{p}^{\mathrm{KPSW}}(1)$ as the fraction estimate for one-step forecast error variance of stock price due to permanent shocks by the KPSW method, and $V_{p}^{\mathrm{CVAR}}(1)$ as that for the CVAR model with $\alpha_{1}=0$ imposed. The null hypothesis of the test is $H_{0}: V_{p}^{\mathrm{KPSW}}(1)-V_{p}^{\mathrm{CVAR}}(1) \leq 0$. The $p$-values of the test are obtained by using bootstrap resampling with 1000 repetitions. For each resampled data, the two estimates are calculated and the bootstrap distribution of the test statistic is obtained. The test results in Table 9 show that the null hypothesis should be rejected at the $1 \%$ level for Sample (D) and Sample (E) and at the 10\% level for Sample (C), that is, the permanent component of the stock price is significantly larger than those estimates using the CVAR with $\alpha_{1}=0$ or from Cochrane (1994).

The above result verifies our analysis in Section 3. If both adjustment coefficients are significant, permanent shocks in the KPSW model are different from that in the CVAR model assuming $\alpha_{1}=0$. In System II, $\alpha_{1} \neq 0$, therefore, the results of the KPSW approach and the CVAR approach with $\alpha_{1}=0$ should be different. The empirical results show that the CVAR approach with $\alpha_{1}=0$ underestimates the permanent shocks in stock price and overestimates the transitory shocks when the weak exogeneity assumption of the dividend for the cointegrating vector that $\alpha_{1}=0$ does not hold.

As we find $\alpha_{1}=0$, the dividend is not a pure random walk. The variation in dividend can be affected by transitory shocks if $\alpha_{1}$ and $\delta_{2}$ are not zero. Our results from the KPSW method show that a substantial amount of the forecast error variance for quarterly 
dividend changes is due to transitory shocks $-21 \%$ for Sample (C), $46 \%$ for Sample (D) and $63 \%$ for Sample (B). These numbers would be zero if the CVAR method with $\alpha_{1}=0$ were used.

\section{Conclusions}

In this paper we use the KPSW method that does not require the assumptions of weak exogeneity used by Cochrane (1994) in order to identify the shocks as permanent and transitory. We show that if one of the error correction coefficients in a bivariate cointegrated system is zero, the CVAR method produces the same type of shocks as the KPSW method. If not, the CVAR method is not a proper method to examine permanent and transitory components of a cointegrated system. The empirical analysis of the consumption-GDP system and dividend-stock price system verifies our theoretical finding.

With both adjustment coefficients significant in the VECM estimate for the consumption-GDP system and for the dividend-stock price system, the permanent shocks for the KPSW method is greater than the CVAR with weak exogeneity assumption. The empirical study shows that for the consumption-GDP system and for the dividend-stock price system, the contribution of the permanent shocks to the forecast error variance of GDP or stock prices may be underestimated if we use the CVAR approach when the weak exogeneity does not hold.

In the CVAR approach with the weak exogeneity assumption, only the consumption shocks or the dividend shocks are permanent shocks for the two systems, respectively. GDP shocks and price shocks are regarded as transitory if there are no consumption or dividend changes. However, sometimes, even with the consumption unchanged, some shocks to GDP may also have permanent effects on GDP. Under such circumstances, the weak exogeneity assumption does not hold. Those shocks are regarded as transitory shocks in the CVAR model, however, in the KPSW approach they are treated as permanent shocks, resulting in the difference in the results.

\section{Acknowledgements}

We would like to thank an anonymous referee for useful comments. Gonzalo acknowledges two grants from the Spanish Ministry of Education (SEJ 200404101 ECON, SEJ 200763098 ECON). Lee thanks the Academic Senate of UC Riverside for financial support. Yang thanks the Chancellor's Fellowship from UC Riverside.

\section{Note}

1. The results in Panel $\mathrm{B}$ and Panel $\mathrm{C}$ are quite similar, indicating we in fact are nearly reproducing Cochrane's results (1994) using our data sets. The small difference is partly due to the estimation of $\beta_{2}$ in Panel B instead of using $\beta_{2}=1$ in Panel C and also partly due to the slightly different data we use from the FRED data for GDP series than the private GNP data of Cochrane (1994).

\section{Notes on contributors}

Jesus Gonzalo is Professor of Econometrics at Universidad Carlos III de Madrid. He received a PhD from University of California, San Diego in 1991. He specializes in econometrics, especially in the areas of nonstationary time series, nonlinear time series modelling, and financial econometrics.

Tae Hwy Lee is Professor of Economics at University of California, Riverside. He received a PhD from University of California, San Diego in 1990. He specializes in econometrics, especially in the areas of nonstationary time series, nonlinear time series modeling, and financial econometrics.

Weiping Yang is an economist at Capital One Financial Services, Richmond, Virginia. He received a PhD from University of California, Riverside in 2005. 


\section{References}

Bansal, Ravi, and Christian Lundblad. 2002. Market efficiency, asset returns and the size of the risk premium in global equity markets. Journal of Econometrics 109, no. 2: 195237.

Barsky, R.B., and B. De Long. 1993. Why does the stock market fluctuate?. Quarterly Journal of Economics 108, no. 2: 291311.

Blanchard, O.J., and D. Quah. 1989. The dynamic effects of aggregate demand and supply disturbances. American Economic Review 79, no. 5: 65573.

Cochrane, J.H. 1994. Permanent and transitory components of GNP and stock prices. Quarterly Journal of Economics 107: 24165.

Crowder, W.J., and M.E. Wohar. 1998. Stock price effects of permanent and transitory shocks. Economic Inquiry 36: 54052.

Engle, R.F., and C.W.J. Granger. 1987. Cointegration and error correction: Representation, estimation, and testing. Econometrica 55: 25176.

Fisher, L., and H. Huh. 2007. Permanent transitory decompositions under weak exogeneity. Econometric Theory 23: 1839.

Fuller, W.A. 1976. Introduction to statistical time series. New York: Wiley.

Gonzalo, J., and C.W.J. Granger. 1995. Estimation of common long memory components in cointegrated systems. Journal of Business Economics and Statistics 13: 2735.

Gonzalo, J., and S. Ng. 2001. A systematic framework for analyzing the dynamic effects of permanent and transitory shocks. Journal of Economic Dynamics and Control 25: 152746.

Jaeger, A. 1992. Does consumption take a random walk? Some evidence from macroeconomic forecasting data. Review of Economics and Statistics 60714.

Johansen, S. 1991. Estimation and hypothesis testing of cointegration vectors in Gaussian vector autoregressive models. Econometrica 59: 155180.

King, R.G., C.I. Plosser, J.H. Stock, and M.W. Watson. 1991. Stochastic trends and economic fluctuations. American Economic Review 81: 81940.

Osterwald Lenum, M. 1992. A note with quantiles of the asymptotic distribution of the maximum likelihood cointegration rank test statistics. Oxford Bulletin of Economics and Statistics 54: 461 72.

Politis, D.N., and J.P. Romano. 1994. The stationary bootstrap. Journal of the American Statistical Association 89: 130313.

Ribba, A. 1997. A note on the equivalence of long run and short run identifying restrictions in cointegrated systems. Economic Letters 56: 2736.

Stock, J.H., and M.W. Watson. 1988. Testing for common trends. Journal of the American Statistical Association 83: 1097107 